\title{
Multiple Unmanned Aerial Vehicle Task Allocation Based on Cloud Mode
}

\author{
Xia Chen and Dehui Wang \\ Shenyang Aerospace University, Automation Department \\ Shenyang, China \\ xiachen1108@163.com,343362886@qq.com
}

\begin{abstract}
For multi- multi-unmanned aerial vehicle (UAV) air combat decision problems, this paper proposes a multi-UAV task allocation method based on cloud model. Firstly, we analyses air combat situation advantage of random and fuzzy information. The cloud model is applied to multi-UAV task allocation. We establish air combat superiority index model based on cloud model. And then through comprehensive cloud generation algorithm, we establish combat situation advantages of comprehensive cloud model based on cloud model and present multi-UAV task assignment method based on comprehensive cloud model. Finally, the simulation verification indicate: To evaluate air combat advantage using cloud model, we can get precise and credible combat situation advantage, thus, we get more scientific and reliable multi-UAV task assignment.
\end{abstract}

Keywords: Multi-UAV, Combat situation advantages, Cloud model, Task assignment

\section{Introduction}

The problem of unmanned aerial vehicle task allocation is always an important research topic of unmanned aerial vehicle technology. In recent ten years, the studying of multi-UAV task allocation problem has made some research achievements[1-3]. Curz J. B.[1] considers the soft killing weapon systems, such as jamming and deception, task allocation optimization model is established, and the particle swarm optimization algorithm is used for solving, the task allocation method is presented. Chen Cheng [2] aims at the issue of cooperating multiple air vehicles mission planning, according to the task types, adopt matrix encoding and heuristic rule to ensure the task sequence of every air vehicle, put forward an improved genetic algorithm. Pongpunwattana A. [3] combines evolutionary algorithm and market theory and set up multi-UAV cooperative mission planning system in dynamic environment, and so on.

In a multi-UAV combat environment, due to the limit the accuracy of airborne sensors and the dynamic change of battlefield and the influence of various interference, uncertain sensory information is often obtained. In recent years, multiUAV task allocation problem based on uncertain perception information cause the attention of some scholars[4-6], with respect to UCAV targets assignment problem with uncertain information, Chen Xia [4] build the mathematical model of UCAV targets assignment with uncertain information and UCAV targets assignment by SMAA (Stochastic Multi-criteria Acceptability Analysis) method based on uncertain information. The authors of [5] present a decision-making method for multi-UAV task allocation problem under uncertain environment. The uncertain indicators of UCAV task assignment are analyzed. Combine subjective and objective weights to determine indicators integrated weights. Combining particle swarm optimization (PSO) algorithm and ranking interval numbers method, the task assignment method 
based on interval-uncertain information was given. The authors of [6] analyze the uncertain information of the actual battlefield, present the problem of multi- combat aerial vehicle (UAV) task allocation in uncertain environment. The model of multiUAV task allocation is established in interval information environment based on the target value gains, target damage indicators and voyage costs indicators. The multiUAV dynamic task allocation method is obtained under uncertain environment by SMAA. The authors of [7] do task assignment study at the theory of dynamic fuzzy set, In order to improve the efficiency of multi- agent dynamic task allocation. The authors of [8] use a decision-maker based on fuzzy neural network to solve the problem of task distribution, propose the method of fuzzy neural network.

However, the study of the uncertainty, the traditional fuzzy set method can only solve the problem of fuzzy target information, and the theory of probability method can only solve the problem of random target information, , but in the multi-UAV combat under uncertain environment, obtained sensory information is usually fuzzy and randomness. For example, the speed of the UAV can be described as a fuzzy situation advantages. Due to the limit the accuracy of airborne sensors and the dynamic change of battlefield and the influence of various interference, The membership degree of the speed advantage has also randomness. Cloud model can combine the fuzziness and randomness and can realize the uncertainty transition between a qualitative concept and its quantitative expression. Currently, related studies based on cloud model cause the attention of some scholars [9-11]. Cloud model [9] has been introduced into the uncertain linguistic multi-criteria group decision-making in order to solve the qualitative and quantitative transformation of the evaluation. The authors of [10] introduce the number characters and relative algorithms of cloud model, and establish the target thread degree judgment rule, and present translation method of qualitative and quantitative evaluation. Aiming at the uncertainty of aerial targets against a background of joint air defense operations, the target threat assessment attributes [11] are expressed using cloud model, which combines fuzziness with randomness. The thesis presents two methods of threat assessment for aerial targets based on cloud model theory starting from two different ideas, that is, logic reasoning and bayesian network.

From the published literature, although at home and abroad, the study of multiUAV mission planning have made many research results now. Many of the technology is still at the exploratory stage. And under complicated environment, the research multi-UAV mission planning based on cloud model has not been reported in literature. Multi-UAV assignment problem under the fuzzy and random information of environment has not been solved. How to establish multi-UAV task allocation model under the fuzzy and random environment, the multi-UAV task allocation method under uncertainty environment is presented, which will be a new research subject. This paper considers the randomness and fuzziness of air combat situation advantage information, establishes a model of air combat superiority index based on cloud model, and establishes air combat situation advantage comprehensive cloud model based on cloud model, presents multi-UAV task assignment method based on cloud model, provides a reliable technical support for multi-UAV air combat decision problem. 


\section{Normal Cloud and its Digital Characteristics}

According to the literature [12], give the representation method of normal cloud and its digital characteristics.

$\mathrm{U}$ is a quantitative universe of discourse (one-dimension or multi-dimension) represented by exact numerical values. $\mathrm{C}$ is the linguistic term relate to $\mathrm{U}$. The membership degree of $\mathrm{x}$ in $\mathrm{U}$ to the linguistic term $\mathrm{C}, \mu(x) \in[0,1]$, is a random number with a stable tendency. A cloud of $\mathrm{C}$ is a mapping from the universe of discourse $\mathrm{U}$ to the unit interval $[0,1]$. That is as follows:

$$
\mu: \mathbf{U} \rightarrow[0,1] \quad \forall x \in \mathbf{U}, x \rightarrow \mu(x)
$$

The distribution of $\mathrm{x}$ in $\mathrm{U}$ is called cloud model, which is briefly called cloud, each $\mathrm{x}$ is called a cloud drop.

A compatibility cloud characterizes the qualitative conception with three digital characters which are respectively expected value Ex, entropy En and hyper entropy He. As shown in Figure 1

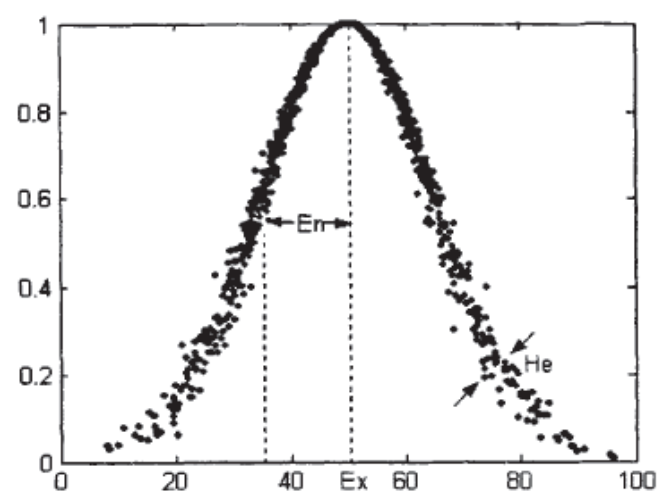

Figure 1. Digital Characteristics of the Cloud

Expectation Ex is the point that cloud represents the qualitative conception properly in the discussion area. This is the most typical sample of the conception.

Entropy En represents a measured granularity of the qualitative conception. Generally, the larger En is, the more macroscopical the conception. Also, En represents the fuzziness of the qualitative conception.

Hyper entropy $\mathrm{He}$ is the uncertain measurement of entropy, namely the entropy of the entropy. It reflects the coagulation of uncertainty of all points which representing the concept in the number domain, namely the coagulation degree of cloud drop. The size of hyper-entropy reflects the discrete degree and thickness of cloud indirectly. The hyper-entropy is decided by the random and fuzziness of entropy.

\section{Combat Situation Attribute Analysis}

In the air, the comprehensive ability of air combat superiority contains airborne survival ability, situation advantage ability and air strikes ability, including our UAV maneuver capability, stealth, electronic self-protection ability, speed advantage, angle advantage, distance advantage, air to air missile missile maneuvering capability, air to air missile missile killing ability, missile guidance capability and other indicators, each an index may have effect on air combat comprehensive advantage. 


\subsection{Air Combat Advantage Comprehensive Ability Evaluation Criteria}

In the air, combat advantage comprehensive ability criteria structure diagram as shown in Figure 2:

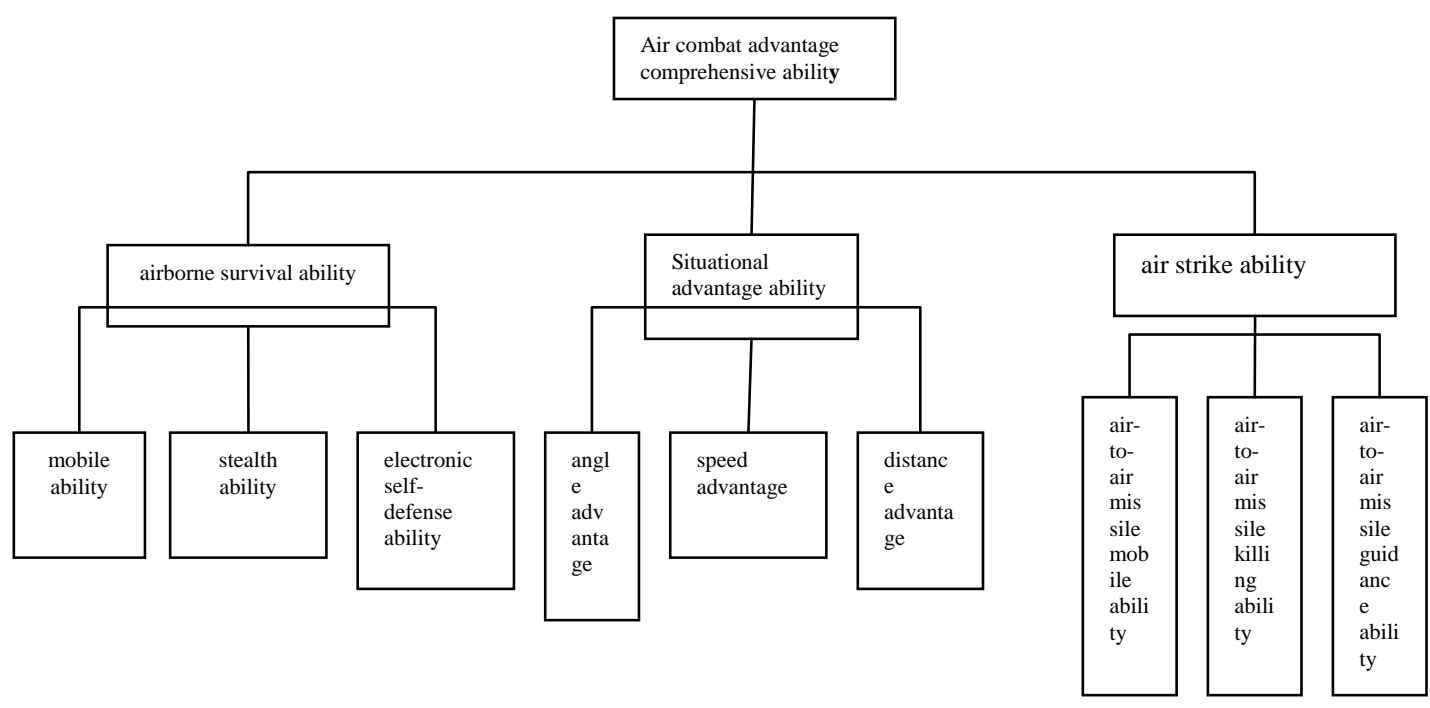

Figure 2. Air Combat Advantage Comprehensive Ability Index Structure Diagram

As we can see from Figure 2, combat advantage comprehensive ability index mainly includes airborne survival ability, situational advantage ability and air strike ability. Airborne viability includes mobile ability, stealth capability and electronic self-defense ability. Situational advantage ability includes angle advantage, speed advantage and distance advantage. Air strike ability includes air-to-air missile mobile ability, air-to-air missile killing ability and air-to-air missile guidance ability.

\subsection{The Establishment of the Air Combat Situation Advantage Function}

Assume our UAV set and enemy set are respectively $\mathrm{M}=\{1,2, \mathrm{~L}, i, \mathrm{~L}, m\}$ and $\mathrm{N}=$ $\{1,2, \mathrm{~L}, j, \mathrm{~L}, n\}$. Set our UAV $i$ againsting enemy $j$ azimuth, entrance the Angle, speed, distance respectively is $\varphi_{i j}, \psi_{i j}, v_{r_{i j}}, D_{i j}$.

\subsubsection{The Angle Advantage Function of our UAV:}

Both our UAV $i$ againsting enemy $j$ the Angle advantage azimuth angle $\varphi_{i j}$ and entrance angle $\psi_{i j}$ influence Angle advantage function, According to the literature [14], the advantage of azimuth Angle:

$$
T_{\varphi_{i j}}=\left\{\begin{array}{l}
0.3\left(1-\frac{\left|\varphi_{i j}\right|-\varphi_{M \text { max }}}{\varphi_{R \text { max }}-\varphi_{M \text { max }}}\right), \varphi_{M \text { max }} \leq\left|\varphi_{i j}\right|<\varphi_{R \text { max }} \\
0.8-\frac{\left|\varphi_{i j}\right|-\varphi_{M K \text { max }}}{2\left(\varphi_{M \text { max }}-\varphi_{M K \text { max }}\right)}, \varphi_{M K \text { max }} \leq\left|\varphi_{i j}\right|<\varphi_{M \text { max }} \\
1-\frac{\left|\varphi_{i j}\right|}{5 \varphi_{M K \text { max }}}, \\
0 \quad, 0 \leq\left|\varphi_{i j}\right|<\varphi_{M K \text { max }}
\end{array}\right.
$$


In the type, $\varphi_{i j}$ is the target azimuth. When it meets $0 \leq\left|\varphi_{i j}\right|<\varphi_{M K \max }$, target in missile cone angle escape is impossible. When it meets $\varphi_{M K \max } \leq\left|\varphi_{i j}\right|<\varphi_{M \max }$, the target is located in the angle of the missile attack area. When it meets $\varphi_{M \max } \leq\left|\varphi_{i j}\right|<\varphi_{R \max }$, the target is located in the airborne radar detecting area.

The influence of target entering angle to advantage:

$$
T_{\psi_{i j}}=\left\{\begin{array}{cl}
\frac{\left|\psi_{i j}\right|}{50^{\circ}} & ,\left|\psi_{i j}\right|<50^{\circ} \\
1-\frac{\left|\psi_{i j}\right|-50^{\circ}}{130^{\circ}} & , 50^{\circ} \leq\left|\psi_{i j}\right| \leq 180^{\circ}
\end{array}\right.
$$

In the type, $\lambda_{1}, \lambda_{2}$ are the weight of the advantage of azimuth Angle and target entering angle respectively. And $\lambda_{1}+\lambda_{2}=1$. So the angle of overall advantage is:

$$
T_{\alpha_{i j}}=\mathrm{T}_{\varphi_{i j}}^{\lambda_{1}} \cdot T_{\psi_{i j}}^{\lambda_{2}}
$$

\subsubsection{Speed Advantage:}

Set our UAV speed is $v_{i}$, and enemy UAV speed is $v_{j} \cdot v_{r_{i j}}=v_{i} / v_{j}$. So speed advantage [14] is

\subsubsection{Distance Advantage:}

$$
T_{v_{i j}}=\left\{\begin{array}{l}
0.1, \quad v_{r_{j j}} \leq 0.6 \\
-0.5+v_{r_{i j}}, 0.6<v_{r_{j}}<1.5 \\
1, \quad v_{r_{j j}} \geq 1.5
\end{array}\right.
$$

The distance of our UAV i againsting enemy $\mathrm{j}$ is ${ }^{D_{i j}}$. The maximum launch range of our UCAV missile is $R_{\max }$. The minimum launch range of our UCAV missile is $R_{\min } \cdot R_{0}=\left(R_{\max }+R_{\min }\right) / 2, \sigma=2\left(R_{\max }-R_{\min }\right)$. The distance advantage is

$$
T_{r_{i j}}=e^{-\left(\frac{D_{i j}-R_{0}}{\sigma}\right)^{2}}
$$

\subsection{Analysis of Air Combat Advantage Attributes Quantification}

Our UAV combat advantage property description includes both quantitative description and the qualitative description. Each attribute value scope is different, physical quantity is not unified.

For the convenience of unified description in future, we quantify the qualitative attribute data first, and then make the quantitative attribute data for reasonable normalization processing, and make all attributes value interval between [0,1]. The greater the attribute value, the bigger the advantage.

According to G.A.Miller 9th quantitative theory[13], In this paper, the quantization processing of our UAV $\mathrm{i}$ air combat advantage the qualitative properties is as follows:

Mobile ability:

$$
t_{i}=\left\{\begin{array}{cc}
0, & \text { no } \\
1 / 2, & \text { medium } \\
1, & \text { strong }
\end{array}\right.
$$

Stealth capability: 


$$
q_{i}=\left\{\begin{array}{cr}
0, & \text { no } \\
1 / 3, & \text { low } \\
2 / 3, & \text { medium } \\
1, & \text { strong }
\end{array}\right.
$$

Electronic self-defense ability

$$
p_{i}=\left\{\begin{array}{cc}
0, & \text { no } \\
1 / 3, & \text { low } \\
2 / 3, & \text { medium } \\
1, & \text { strong }
\end{array}\right.
$$

Air-to-air missile mobile ability

$$
l_{i}=\left\{\begin{array}{cc}
0, & \text { no } \\
1 / 3, & \text { low } \\
2 / 3, & \text { medium } \\
1, & \text { strong }
\end{array}\right.
$$

Air-to-air missile killing ability

$$
e_{i}=\left\{\begin{array}{cc}
0, & \text { no } \\
1 / 3, & \text { low } \\
2 / 3, & \text { medium } \\
1, & \text { strong }
\end{array}\right.
$$

Air-to-air missile guidance ability

$$
u_{i}=\left\{\begin{array}{cc}
0, & \text { no } \\
1 / 3, & \text { low } \\
2 / 3, & \text { medium } \\
1, & \text { strong }
\end{array}\right.
$$

\section{Multi-UAVs Task Allocation Based on Cloud Model}

\subsection{Cloud Model Transformation}

The electronic self-protection ability as an example, convert it to cloud model, other properties similar to it. Our UAV's electronics self-protection ability divide into no, low, medium and strong, quantize them to $0 、 1 / 3 、 2 / 3 、 1$, in turn. That is to say, $E x(i, j)$ are respectively $0 、 1 / 3 、 2 / 3 、 1$. Because the normal distribution is a special case of the normal cloud when $\mathrm{He}=0$, In a normal cloud, [Ex-3En, Ex+3En] Range of cloud has contributed $99.74 \%$ of the entire cloud (the so-called

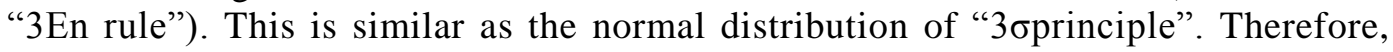
for distinguishing each cloud better, we ensure all the advantage of cloud in the corresponding field. Cloud theory of entropy En set to 1/6 of the width of the domain, Cloud of hyper entropy He decided jointly by entropy of randomness and fuzziness, as a matter of experience [16], $\mathrm{He}=\mathrm{En} / 10$. According to the literature [11], Entropy and hyper entropy respectively is: $E n(i, j)=1 / 18, \quad H e(i, j)=1 / 180$. 


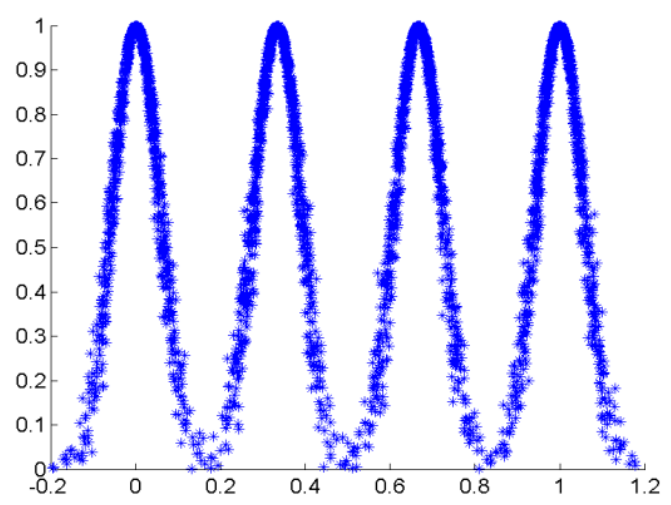

Figure 3. Our UAVs Electronic Self-defense Ability Cloud Model

The speed advantage as an example, is converted to cloud model, is similar to Angle advantage and distance advantage. When the speed of the UAV is 0.8 , the cloud model is shown in figure 4 at this time.

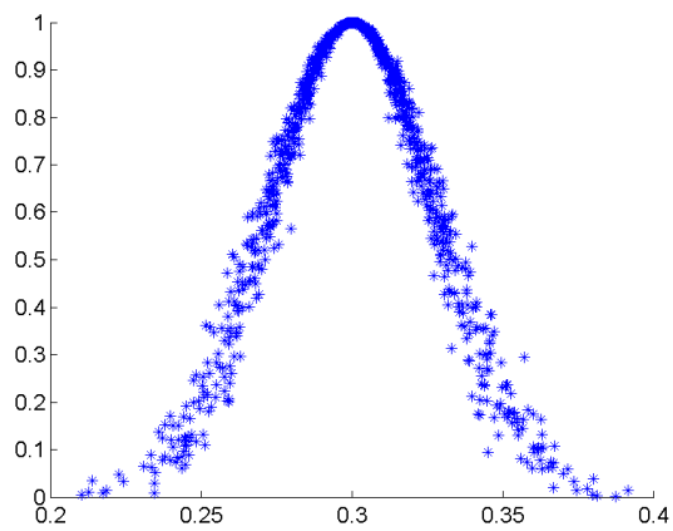

Figure 4. The Cloud Model is that the Speed of the UAV is 0.8

\subsection{Comprehensive Cloud Generation Algorithm}

In Figure 2, each index attribute value of air combat advantage comprehensive ability can be represented by a cloud model, so $\mathrm{n}$ index attribute value (cloud model) can be expressed as a comprehensive cloud.

$$
\begin{aligned}
& E x(i, j)=\omega_{1} E x_{1}(i, j)+\omega_{2} E x_{2}(i, j)+\mathrm{L}+\omega_{n} E x_{n}(i, j) \\
& E n=\frac{\omega_{1} E x_{1} E n_{1}+\omega_{2} E x_{2} E n_{2}+\mathrm{L}+\omega_{n} E x_{n} E n_{\mathrm{n}}}{\omega_{1} E x_{1}+\omega_{2} E x_{2}+\mathrm{L}+\omega_{n} E x_{n}} \\
& H e=E n / 10
\end{aligned}
$$

In this type, $\omega_{1}+\omega_{2}+\mathrm{L}+\omega_{n}=1$

\subsection{Task Allocation Based on Cloud Picture}

In the multi-UAV multi-target combat task allocation process, first of all, according to the uncertain fuzzy random situation awareness information, cloud model of each attribute index is generated. Then, according to the comprehensive cloud generation algorithm, the comprehensive cloud of air combat advantage comprehensive ability is generated. 
The expectation Ex signify the mean of comprehensive advantages. The bigger the expectation Ex is, the greater the UAV comprehensive situational advantages are. So we assign task according to the size of the expectations Ex, firstly.

If the expectations Ex is the same, we assign task according to ascending order of the entropy En. Entropy En signify discrete degree of air combat advantage comprehensive ability, it reflects cloud droplets randomness of air combat advantage the comprehensive ability. At the same time, it also reflects its comprehensive ability fuzziness and the scope of their comprehensive ability cloud droplets.

The smaller entropy En, the less the randomness and fuzziness of comprehensive situational advantage ability, the stability of the comprehensive advantage ability is greater. If the expectation Ex and entropy En are all the same, we allocate the task according to smallest hyper entropy. Hyper entropy $\mathrm{He}$ is the uncertain measurement of entropy, namely the entropy of the entropy. It reflects the discrete degree of air combat advantage comprehensive ability of cloud droplets. The size of hyper entropy indirectly reflects the thickness of the cloud of the air combat advantage comprehensive ability. The bigger hyper entropy is, the thicker the cloud thickness is.

\subsection{The Main Steps of Algorithm}

Step1: according to the type (1)-(3), calculate our UAV $i$ againsting enemy $j$ the Angle advantage $T_{\alpha_{i j}}$, speed advantage $T_{v_{i j}}$, speed advantage $T_{r_{i j}}$ respectively.

Step2: according to the figure 2 and the type (4)-(9), make quantitative processing for our UAV $i$ air combat advantage attributes, mobile ability $t_{i}$, stealth capability $q_{i}$, electronic self-defense ability $p_{i}$, air-to-air missile mobile ability $l_{i}$, air-to-air missile killing ability $e_{i}$, air-to-air missile guidance ability $u_{i}$.

Step3: based on cloud model conversion method, establish air combat advantage ability index of our UAV $i$ againsting enemy $j$, the Angle advantage $T_{\alpha_{i j}}$ cloud model, speed advantage $T_{v_{i j}}$ cloud model, speed advantage $T_{r_{r_{j}}}$ cloud model, mobile ability $t_{i}$ cloud model, stealth capability $q_{i}$ cloud model, electronic self-defense ability $p_{i}$ cloud model, air-to-air missile mobile ability $l_{i}$ cloud model, air-to-air missile killing ability $e_{i}$ cloud model, air-to-air missile guidance ability $u_{i}$ cloud model.

Step4: according to the type (10)-(12) and the given weight $\omega_{k}$, establish our UAV $i$ againsting enemy $j$ air combat advantage comprehensive abilities cloud model, in this, $i=1,2, \mathrm{~L}, m, j=1,2, \mathrm{~L}, n$.

Step5: according to our UAV $i$ againsting enemy $j$ air combat advantage comprehensive abilities cloud model $E x(i, j), i=1,2, \mathrm{~L}, m, j=1,2, \mathrm{~L}, n$, calculate $\max _{1 \leq i \leq m} E x(i, j)=E x\left(i_{1}^{*}, j_{1}^{*}\right)$, so the target $j_{1}^{*}$ will be assigned to our UAV $i_{1}^{*}$. If our UAV's $\underset{\substack{1 \leq i \leq m \\ 1 \leq j \leq n}}{\sin (x)}$

expectations for the same target is same, that is $\max _{\substack{1<i<m \\ 1<\leqslant}} E x(i, j)=E x\left(i_{1}^{*}, j_{1}^{*}\right)=E x\left(i_{1}^{*}, j_{1}^{*}\right)$, $\underset{\substack{1 \leq i \leq m \\ 1 \leq j \leq n}}{1 j \leq x}$

respectively calculate its the entropy $E n\left(i_{1}^{*}, j_{1}^{*}\right)$ and $E n\left(i_{1}^{*}, j_{1}^{*}\right)$. If $E n\left(i_{1}^{*}, j_{1}^{*}\right)>E n\left(i_{1}^{*}, j_{1}^{*}\right)$, the target $j_{1}^{*}$ will be assigned to our UAV $i_{1}^{*}$. And the target $j_{1}^{*}$ is removed.

Step6: in the rest of the targets, continue to the second selection $\max _{1 \leq i \leq m} E x(i, j)=E x\left(i_{2}^{*}, j_{2}^{*}\right)$, in this, $i=1,2, \mathrm{~L}, m, j=1,2, \mathrm{~L}, n-1$, the target $j_{2}^{*}$ will be $1 \leq i \leq m$
$1 \leq j \leq n-1$

assigned to our UAV $i_{2}^{*}$. If our UAV's expectations for the same target is same, that 
is $\max E x(i, j)=E x\left(i_{2}^{*}, j_{2}^{*}\right)=E x\left(i_{2}^{*}, j_{2}^{*}\right)$, respectively calculate its the entropy $E n\left(i_{2}^{*}, j_{2}^{*}\right)$ $\underset{\substack{1 \leq i \leq m \\ 1 \leq j \leq n}}{1 \leq j}$

and $E n\left(i_{2}^{*}, j_{2}^{*}\right)$. If $E n\left(i_{2}^{*}, j_{2}^{*}\right)>E n\left(i_{2}^{*}, j_{2}^{*}\right)$, the target $j_{2}^{*}$ will be assigned to our UAV $i_{2}^{*}$. And the target $j_{2}^{*}$ is removed.

Step7: repeat step 6, until all the $\operatorname{targets}(j=1,2, \mathrm{~L}, n)$ are assigned.

\section{Simulation}

In this paper, considering two drones against. The performance of the airborne radar searching azimuth $\varphi_{R \max }= \pm 120^{\circ}$, the performance for air-to-air missile : The maximum off-axis emission angle $\varphi_{M \max }= \pm 80^{\circ}$, cone angle $\varphi_{M K \text { max }}= \pm 30^{\circ}$. Our drones and enemy air combat situation information is as follows:

Table 1. The Azimuth Angle $\varphi_{i j}{ }^{\circ}$ ) Information of UAV

\begin{tabular}{ccc}
\hline & Target 1 & Target 2 \\
\hline UAV 1 & $8^{\circ}$ & $20^{\circ}$ \\
UAV 2 & $26^{\circ}$ & $9^{\circ}$ \\
\hline
\end{tabular}

Table 2. The Entering Angle $\psi_{i j}{ }^{\circ}$ ) Information of UAV

\begin{tabular}{ccc}
\hline & Target 1 & Target 2 \\
\hline UAV 1 & $55^{\circ}$ & $10^{\circ}$ \\
UAV 2 & $150^{\circ}$ & $57^{\circ}$ \\
\hline
\end{tabular}

Table 3. The Speed $v_{r_{j}}=v_{i} / v_{j}$ Information of UAV

\begin{tabular}{ccc}
\hline & Target 1 & Target 2 \\
\hline UAV 1 & 1.6 & 0.6 \\
UAV 2 & 1.5 & 1.4 \\
\hline
\end{tabular}

Table 4. The distance $D_{i j}(\mathrm{~km})$ Information of UAV

\begin{tabular}{ccc}
\hline & Target 1 & Target 2 \\
\hline UAV 1 & 25 & 60 \\
UAV 2 & 75 & 26 \\
\hline
\end{tabular}

Table 5. Other Information of UAV

\begin{tabular}{|c|c|c|c|c|c|c|}
\hline UAV 1 & $\begin{array}{r}\text { mobile } \\
\text { ability } t_{i} \\
\text { medium }\end{array}$ & $\begin{array}{c}\text { stealth } \\
\text { capability } q_{i} \\
\text { low }\end{array}$ & $\begin{array}{c}\text { electronic self-defense } \\
\text { ability } p_{i} \\
\text { low }\end{array}$ & $\begin{array}{c}\text { air-to-air missile } \\
\text { mobile ability } l_{i} \\
\text { low }\end{array}$ & $\begin{array}{c}\text { air-to-air missile killing } \\
\text { ability } e_{i} \\
\text { low }\end{array}$ & $\begin{array}{c}\text { air-to-air missile } \\
\text { guidance ability } u_{i} \\
\text { medium }\end{array}$ \\
\hline UAV 2 & medium & medium & medium & strong & medium & strong \\
\hline
\end{tabular}

According to the step 1 to step 3, it is concluded that combat situation advantages integrated cloud is shown in figure 5. 


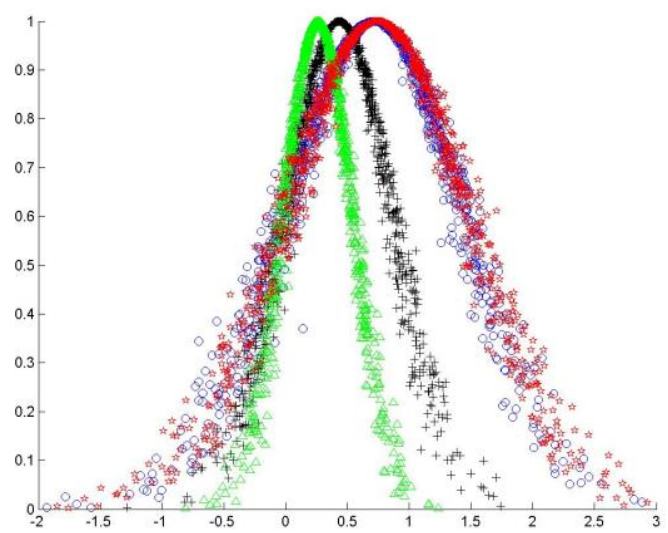

\section{Figure 5. Air Combat Advantage Cloud Picture}

In the figure, The blue circle is advantage of the cloud that UAV1 relative to target 1.Black plus is advantage of the cloud that UAV1 relative to target 2.green triangle is advantage of the cloud that UAV2 relative to target 1.Red star is advantage of the cloud that UAV2 relative to target 1 .

By the figure 5, we can see that the air combat advantage of our UAV1 attacking target 1 and our UAV2 attacking target 2 are maximum, so the result of task allocation is that our UAV1 attacks target 1 and our UAV2 attacks target2.

\section{Conclusion}

In air combat decision, Studying multi-UAVs multi-target assignment problem on uncertain environment is an important research topic in the air combat situation. Through the advantages of information ofrandom and fuzzy, this paper presents for multi-UAVs task assignment method based on cloud model. We build air combat superiority index model based on cloud model, and establish combat situation advantages of comprehensive cloud model based on cloud, present multi-UAVs task assignment method based on cloud model. And the feasibility and effectiveness of this method was verified by simulation. In order to solve multi-UAVs task assignment problem under complex environment, provides a more scientific method. which can provide a reliable technical support for further study under the uncertain environment of multi-UAVs combat problem.

\section{References}

[1] J. B. Curz, C. Jr. and G. Chen, "Particle Swarm Optimization for Resource Allocation in UAV Cooperative Control", AIAA Guidance Navigation, and Control Conference and Exhibit, (2004).

[2] C. Chen, L. N. Xing and J. Y. Tan, "Improved Genetic Algorithm for Cooperating Multi Air Vehicle Mission Planning", Ordnance Industry Automation, vol.29, (2010), pp.28-31.

[3] A. Pongpunwattana and R. Rysdyk, "Real-Time Planning for Multiple Autonomous Vehicles in Dynamic Uncertain Environments", Journal of Aerospace Computing, Information, and Communication, vol.1, (2004), pp.580-605.

[4] X. Chen and Z. G. Guo, "UCAV Targets Assignment Method Based on Uncertain Information", Journal of System Simulation, vol.24, (2012), pp.1651-1654.

[5] X. Chen and Y. X. Hu, "Task Assignment of Multi-UCAV Based on PSO Algorithm Under Uncertain Environment", Ordnance Industry Automation, vol.32, (2013), pp.11-16.

[6] X. Chen and T. Tang, "Study on the Dynamic Task Assignment of Multi- UCAV under Dynamic Uncertain Environment”, Fire Control \& Command Control, vol.29, (2013), pp.119-122.

[7] F. Yin and L. Niu, "Multiple Agent Dynamic Task Allocation Algorithm Research Based on Dynamic Fuzzy Sets", Bulletin of Science and Technology, vol.29, (2013), pp.119-122. 
[8] H. Dong and H. S. Yan, "Decision of task distribution in manufacturing system oriented agent based on fuzzy neural network", Manufacturing Automation, vol.25, (2003), pp.14-18.

[9] J. Q. Wang and T. Liu, "Based on cloud model of uncertain linguistic multiple criteria decision-making method and application research", (2011).

[10] X. X. Wang and W. M. Wang, "Air-attack Target Threat Assessment Based on Cloud Mode", Computer \& Digital Engineerin, vol.2, (2013), pp.41-43.

[11] Y. Y. Zhang, "Methods of Threat Assessment for Aerial Targets based on Cloud Model Theory", (2013).

[12] X. Y. Du, Q. J. Yin, K. D. Huang and D. N. Liang, "Transformation between qualitative variables and quantity based on cloud models and its application", Systems Engineering and Electronics, vol.30, (2008), pp.772-776.

[13] O. Kulak, "A decision support system for fuzzy multi-attribute selection of material handling equipments", Expert Systems with Applications, vol.29, (2005), pp.310-319.

[14] Y. Gao and J. W. Xiang, "New Threat Assessment Non-Parameter Model in Beyond-Visual-Range Air Combat", Journal of System Simulation, vol.18, (2006), pp.2570-2572.

[15] D. Y. Li, C. Y. Liu, Y. Du and X. Han, "Artificial Intelligence with Uncertainty", vol.15, (2005), pp.1584-1594.

[16] S. X. Wang, L. Zhang, S. Wang, J. F. Shen and Y. Liu, "Qualitative and Quantitative Representing and Reasoning for Goals Satisfiability”, Journal of Software, vol.22, (2011), pp.593-608.

\section{Authors}

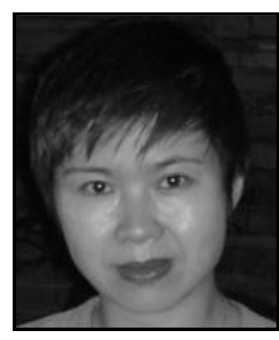

Xia Chen, she was born on 1962 and she is a professor in Shenyang Aerospace University. Her main research interests are measurement technique, automatic control, flight control, etc.

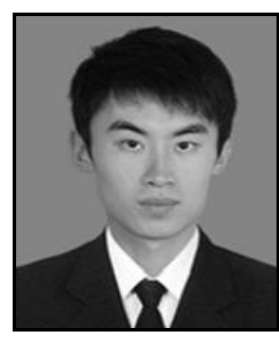

Dehui Wang, he was born on 1989 and he is a graduate student in Shenyang Aerospace University. His main research interest is the research of unmanned aerial vehicle (UAV) task allocation. 
International Journal of Control and Automation Vol. 8, No. 9 (2015) 